\title{
Ideals and some applications of simply open sets
}

\author{
Arafa A. Nasef ${ }^{a}$ and R. Mareay ${ }^{b, *}$ \\ ${ }^{a}$ Department of Physics and Engineering Mathematics, Faculty of Engineering, Kafrelsheikh University, \\ ${ }^{b}$ Department of Mathematics, Faculty of Science, Kafrelsheikh University, \\ Kafr El-Sheikh 33516, Egypt.
}

\section{ABSTRACT}

Recently there has been some interest in the notion of a locally closed subset of a topological space. In this paper, we introduce a useful characterizations of simply open sets in terms of the ideal of nowhere dense set. Also, we study a new notion of functions in topological spaces known as dual simply-continuous functions and some of their fundamental properties are investigated. Finally, a new type of simply open sets is introduced.

Keywords and phrases: Ideal; simply open sets; simply continuous; strongly simply continuous and dual simply continuous functions.

\section{Introduction}

According to Biswas [4] and Neubrunnovaá[23], a subset $B$ of a space $(X, \tau)$ is called simply open if it is the union of an open set and a nowhere dense set. In 1969 Biswas [4]introduced the concept of simply continuity and introduced some of its properties. Also, Ewert and Neubrunnova' used simply open set in [13] and [23] to define the concept of simply continuity, i.e. a function $f: X \rightarrow Y$ is simply continuous if the inverse image with respect to $f$ of any open set in $Y$ is simply open in $X$. Also, Dontchev and Ganster [11] used simply open sets to define the concept of strongly simply continuity, i.e., a function $f: X \rightarrow Y$ is strongly simply continuous if the inverse with respect to $f$ of any semi-open set in $Y$ is simply open in $X$. This enabled them to produce a decomposition of continuity for functions between arbitrary topological spaces.

Let $(X, \tau)$ be a topological space. For a subset $B$ of $X$, the closure and the interior of $B$ with respect to $(X, \tau)$ will be denoted by $C l(B)$ and $\operatorname{Int}(B)$, respectively. This paper provides a useful characterizations of simply open sets in terms of the ideal of nowhere dense set. Also, we introduce and study a new notion of functions in topological spaces known as dual simply-continuous functions and investigate some of their fundamental properties.

\section{2 preliminaries}

Definition 2.1 A subset $A$ of a topological space $(X, \tau)$ is called:

1. semi-open [18] if $A \subseteq C l(\operatorname{Int}(A))$,

2. semi-closed [9] if $X \backslash A$ is semi-open,or equivalently, if $\operatorname{Int}(\operatorname{Cl}(A)) \subseteq A$.

3. an $\alpha$-set or $\alpha$-open [24] if $A \subseteq \operatorname{Int}(\operatorname{Cl}(\operatorname{Int}(A)))$,

4. $\alpha$-closed [24] if $X \backslash A$ is $\alpha$-open, or equivalently, if $C l(\operatorname{Int}(C l(A))) \subseteq A$,

5. preopen [21] if $A \subseteq \operatorname{Int}(C l(A))$,

6. nowhere dense if $\operatorname{Int}(C l(A))=\varnothing$,

7. regular open [26] if $A=C l(\operatorname{Int}(A))$.

The collection of semi -open sets, semi -closed sets and $\alpha$-sets in $(X, \tau)$ will be denoted by $\operatorname{SO}(X, \tau)$, $S C(X, \tau)$ and $\tau^{\alpha}$, respectively. Njåstad [24] has shown that $\tau^{\alpha}$ is a topology on $X$ with the following properties: $\tau \subseteq \tau^{\alpha},\left(\tau^{\alpha}\right)^{\alpha}=\tau^{\alpha}$ and $A \in \tau^{\alpha}$ if and only if $A=U \backslash N$ where $U \in \tau$ and $N$ is nowhere dense in $(X, \tau)$. Hence $\tau=\tau^{\alpha}$ if and only if every nowhere dense set in $(X, \tau)$ is closed. Clearly every $\alpha$-set is semi-open and every nowhere dense set in $(X, \tau)$ is semi-closed. Andrijevi'c [2] has observed that $S O\left(X, \tau^{\alpha}\right)=S O(X, \tau)$ and that $N \subseteq X$ is nowhere dense in $\left(X, \tau^{\alpha}\right)$ if and only if $N$ is nowhere dense in $(X, \tau)$.

Definition 2.2 A subset $A$ of a topological space $(X, \tau)$ is called: 
1. $\delta$-set [8] if $\operatorname{Int}(C l(A)) \subseteq C l(\operatorname{Int}(A))$,

2. $s e m i$-locally closed [28] if $A$ is the intersection of a semi-open set and a semi-closed set,

3. $N D B$-set [10] if the boundary of $A$ is nowhere dense,

4. $s g$-closed [3] if the semi-closure of $A$ is included in every semi-open superset of $A$,

5. locally closed [6] if $A=G \cap F$ where $G$ is open and $F$ is closed, or, equivalently, if $A=G \cap C l(A)$ for some open set $U$.

We will denote the collections of all locally closed sets and semi-locally closed sets of $(X, \tau)$ by $L C(X, \tau)$ and $\operatorname{SLC}(X, \tau)$, respectively. Note that Stone [27] has used the term FG for a locally closed subset. A dense subset of $(X, \tau)$ is locally closed if and only if it is open.

Definition 2.3 Recall that a function $f:(X, \tau) \rightarrow(Y, \sigma)$ is called:

1. irresolute [9] if $f^{-1}(V)$ is semi-open in $(X, \tau)$ for every semi-open set $V$ of $(Y, \sigma)$;

2. semi-continuous [18] if $f^{-1}(V)$ is semi-open in $(X, \tau)$ for every open set $V$ of $(Y, \sigma)$;

3. strongly semi-continuous [1], if $f^{-1}(V)$ is open in $(X, \tau)$ for every semi-open set $V$ of $(Y, \sigma)$;

4. simply continuous $[4,13,23]$, if $f^{-1}(V)$ is simply-open in $(X, \tau)$ for every open set $V$ of $(Y, \sigma)$.

5. strongly simply-continuous [11], if for every semi-open set $V$ of $Y, f^{-1}(V)$ is simply-open in $X$;

6. pre $s g$-continous [25] if $f^{-1}(V)$ is $s g$-closed in $(X, \tau)$ for every semi-closed set $V$ of $(V, \sigma)$.

\section{Simply-open sets}

Definition 3.1 [4, 23]. A subset $B$ of a topological space $(X, \tau)$ is called simply-open if $B=G \cup N$, where $G$ is an open set and $N$ is nowhere dense in $(X, \tau)$.

By [4], the union and the intersection of two simply open sets is a simply open sets, the complement of a simply open set is a simply open set.

The following proposition is a slight enlargement of Theorem 2.2 from [15].

Proposition 3.1 For a subset $B \subseteq(X, \tau)$ the following conditions are equivalent:

1. $B$ is simply-open.

2. $\operatorname{Fr}(B)$ (where $\operatorname{Fr}(B)=C l(B) \backslash \operatorname{Int}(B))$ is nowhere dense in $X$

3. there exist two subsets $G$ and $H$ of $X$ where $G$ is open and $H$ is nowhere dense in $X$, such that $G \cup H \subseteq B \subseteq C l(G \cup H)$

4. $B$ is semi-locally closed.

5. $B$ is a $\delta$-set.

6. $B$ is an $N D B$-set.

7. $B \in L C\left(X, \tau^{\alpha}\right)$.

Proof. $(1) \Leftrightarrow(2)$ : (see [[4],Remark 1])

$(1) \Leftrightarrow(3):(\operatorname{see}[r 4$, Definition 1$])$ 
The implications $(1) \Leftrightarrow(4) \Leftrightarrow(5)$ is given in [15].

$(5) \Leftrightarrow(6)$ : Follows from the identity: $\operatorname{Int}(\operatorname{Fr}(B))=\operatorname{Int}(C l(B)) \cap \operatorname{Int}(C l(X \backslash B))$

$$
\begin{aligned}
& =\operatorname{Int}(C l(B)) \cap(X \backslash C l(\operatorname{Int}(B))) \\
& =\operatorname{Int}(C l(B)) \backslash C l(\operatorname{Int}(B)) .
\end{aligned}
$$

Remark 3.1 One can deduce that:

$$
\text { open set } \Rightarrow \text { semi-open set } \Rightarrow \text { simply-open set }
$$

Clearly every semi-open and every semi-closed set is simply-open. Conversely, not every simply-open set is semi-open or semi-closed. As shown by the following example.

Example 3.1 Consider the following subset of the real line with the usual topology: $S=\left(0, \frac{1}{2}\right) \cup\left(\frac{1}{2}, 1\right) \cup\{2\}$. Dontchev and Ganster [11] proved that $S$ is simply-open but neither semi-open nor semi-closed.

\section{Proposition 3.2}

1. The family of all simply-open sets in a topological space $(X, \tau)$ is an algebra of sets, i.e. it contains the complement of each member as well as the union of each two members.

2. The finite intersection of simply-open sets is also simply-open.

Proposition 3.3 [11] For a subset $B \subseteq(X, \tau)$, the following conditions are equivalent:

1. $B$ is semi-closed.

2. $B$ is $s g$-closed and simply-open.

Proof. (1) $\Rightarrow(2)$ : is clear.

$(2) \Rightarrow(1)$ : since $B$ is simply-open, then $B$ can be written as the intersection of a semi-open set $S$ and a semi-closed set $F$. Since $B$ is $s g$-closed, we have that $S C l(B)$ is contained in $S$. Since $F$ is semi-closed, $S C l(B)$ is contained in $F$. Therefore, $S C l(B)=B$, that is $B$ is semi-closed.

Proposition 3.4 For a topological space $(X, \tau)$ the following conditions are equivalent:

1. Every simply-open set is semi-closed,

2. Every open set is regular open,

3. $X$ is locally indiscrete (i.e. every open set is closed),

4. Every simply-open set is $\alpha$-closed.

Proof. $(1) \Rightarrow(2)$ : is in Proposition 2.6 [11].

$(2) \Rightarrow(3)$ : is in Theorem 3.3 from [16].

(3) $\Rightarrow(4)$ : Let $B \in S M O(X)$, i.e. let $B=G \cup N$, where $G$ is open and $N$ is nowhere dense. By (3) , $G$ is closed and hence $\alpha$-closed. Since $N$ is also $\alpha$-closed and since the $\alpha$-open sets form a topology in $X$, then $B$ is $\alpha$-closed as well.

$$
(4) \Rightarrow(1) \text { : is obvious. }
$$

In a topological space $(X, \tau)$, a subset $B$ is a $V_{s}-$ set [7] of $(X, \tau)$ if $B=B^{V_{s}}$, where $B^{V_{s}}=\cup\left\{F: F \subseteq B, F^{c} \in S O(X, \tau)\right\}$. A topological space $(X, \tau)$ is called a semi- $R_{0}-$ space [19] if every semi-open set contains the semi-closure of each of its singletons.

Theorem 3.1 For a topological space $(X, \tau)$ the following conditions are equivalent: 
1. Every simply-open subspace is a $V_{s}$ - set,

2. $(X, \tau)$ is a semi- $R_{0}-$ space,

3. Every open subspace is a $V_{s}$ - set.

Proof. From Remark 2.2, (1) $\Rightarrow(2)$ and (2) $\Rightarrow$ (3) are obvious.

(3) $\Rightarrow$ (1) Let $B \subseteq S M O(X, \tau)$, then $B=U \cup N$, where $U \in \tau$ and $N$ is nowhere dense. By (3), $U$ is a $V_{s}$ - set. Since every nowhere dense set is semi-closed, then by Proposition 3.5. [7] $B$ is a $V_{s}-$ set.

Let $(X, \tau)$ be a topological space and let us denote by $I_{n}$ the ideal of nowhere dense subsets of $(X, \tau)$. On page 69 in [17] Kuratowski defined a subset $A \subseteq X$ to be open mod $I_{n}$ if there exists an open set $G$ such that $A \backslash G \in I_{n}$ and $G \backslash A \in I_{n}$.

Proposition 3.5 (see page 69 in [17])Let $I_{n}$ denote the ideal of nowhere dense sets in a space $(X, \tau)$. Then

1. open sets are open mod $I_{n}$;

2. closed sets are open mod $I_{n}$;

3. If $A, B$ are open mod $I_{n}$, then $A \cap B, A \cup B$ and $X \backslash A$ are open mod $I_{n}$;

4. $A \subseteq X$ is open mod $I_{n}$ if and only if $A=G \cup N$ where $G$ is open and $N$ is nowhere dense in $(X, \tau)$ if and only if $A$ is simply open.

Theorem 3.2 Let $A$ be a subset of a space $(X, \tau)$ and let $I_{n}$ denote the ideal of nowhere dense subsets of $(X, \tau)$. Then the following are equivalent:

1. $A \in L C\left(X, \tau^{\alpha}\right)$;

2. $A \in S L C(X, \tau)$;

3. $A$ is a $\delta-$ set;

4. $A \in \operatorname{SMO}(X, \tau)$;

5. $A$ is open $\bmod I_{n}$.

Proof. $(1) \Rightarrow(2)$ : Follows from the observation that every $\alpha-$ set is semi-open.

(2) $\Rightarrow(3)$ : Let $A \in S L C(X, \tau)$, i.e. $A=G \cap F$ where $G \in S O(X, \tau)$ and $F \in S C(X, \tau)$, i.e. $G \subseteq C l(\operatorname{Int}(G)) \quad$ and $\quad \operatorname{Int}(C l(F)) \subseteq F \quad$. Since $\quad \operatorname{Int}(C l(A)) \subseteq \operatorname{Int}(C l(F)) \subseteq F \quad$, we have $\operatorname{Int}(C l(A)) \subseteq \operatorname{In}(F)$. Since $A \subseteq G \subseteq C l(\operatorname{Int}(G))$ we have $\operatorname{Int}(C l(A)) \subseteq C l(\operatorname{Int}(G))$. Consequently, $\operatorname{Int}(C l(A)) \subseteq C l(\operatorname{Int}(G)) \cap \operatorname{Int}(F) \subseteq C l(\operatorname{Int}(G) \cap \operatorname{Int}(F))=\operatorname{Cl}(\operatorname{Int}(A))$. Hence $A$ is a $\delta-$ set.

$(3) \Rightarrow(4)$ : Assume that $\operatorname{Int}(C l(A)) \subseteq C l(\operatorname{Int}(A))$ and let $U=\operatorname{Int}(A)$ and $N=A \backslash \operatorname{Int}(A)$. We will show that $N$ is nowhere dense. Clearly $\operatorname{Int}(\operatorname{Cl}(N)) \subseteq \operatorname{Int}(C l(A))$, and since $N \cap \operatorname{Int}(A)=\varnothing$, we have $\operatorname{Int}(C l(N)) \cap C l(\operatorname{Int}(A))=\varnothing$. So $\operatorname{Int}(C l(N))=\varnothing$, i.e. $N$ is nowhere dense.

$(4) \Rightarrow(5)$ : See Proposition 2.1 [15].

$(5) \Rightarrow(1)$ : Let $A$ be open $\bmod I_{n}$. By Proposition 2.1 [15], $X \backslash A$ is open $\bmod I_{n}$, so $X \backslash A=U \cup N$ where $U$ is open and $N$ is nowhere dense in $(X, \tau)$. Hence 
$A=(X \backslash A) \cap(X \backslash U) \in L C\left(X, \tau^{\alpha}\right)$ since $X \backslash N \in \tau^{\alpha}$ and $X \backslash U$ is closed in $(X, \tau)$ and consequently closed in $\left(X, \tau^{\alpha}\right)$.

\section{On simply continuous and dual simply continuous functions}

Definition 4.1 A function $f:(X, \tau) \rightarrow(Y, \sigma)$ is called dual simply-continuous if for every simply open set $V$ of $Y, f^{-1}(V)$ is open in $X$.

Proposition 4.1 For a function $f:(X, \tau) \rightarrow(Y, \sigma)$, the following conditions are equivalent:

1. $f$ is simply-continuous;

2. For every closed set $V$ of $Y, f^{-1}(V)$ is simply-open in $X$.

Proposition 4.2 For a function $f:(X, \tau) \rightarrow(Y, \sigma)$, the following conditions are equivalent:

1. $f$ is strongly simply-continuous ;

2. For every semi-closed set $V$ of $Y, f^{-1}(V)$ is simply-open in $X$.

In 1991, Foran and Liebnitz [14] defined a topological space $(X, \tau)$ to be strongly irresolvable if no non-empty open set is resolvable or equivalently if every subset of $X$ is simply-open. In 1969, El'kin [12] defined a topological space $(X, \tau)$ to be globally disconnected if every set which can be placed between an open set and its closure is open, i.e. if every semi-open set is open. A semi-door space [29] is a topological space in which every set is either semi-open or semi-closed. Note that a semi-door space is always strongly irresolvable. The relationships between simply-continuous, dual simply-continuous, strongly simply-continuous and other corresponding types of functions are shown in the following diagram 1:

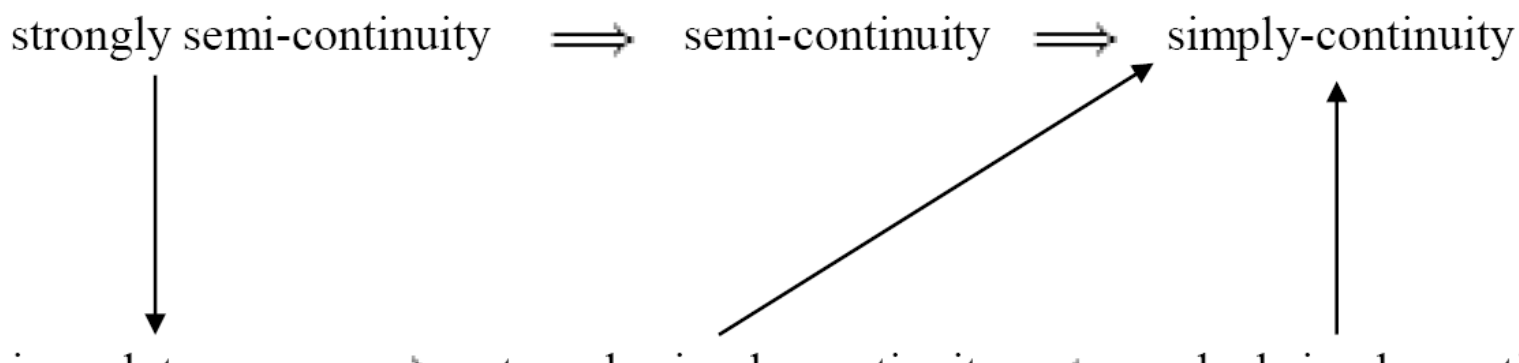

irresoluteness $\Longrightarrow$ strongly simply-continuity $\Longleftarrow$ dual simply-continuity

Figure 1:

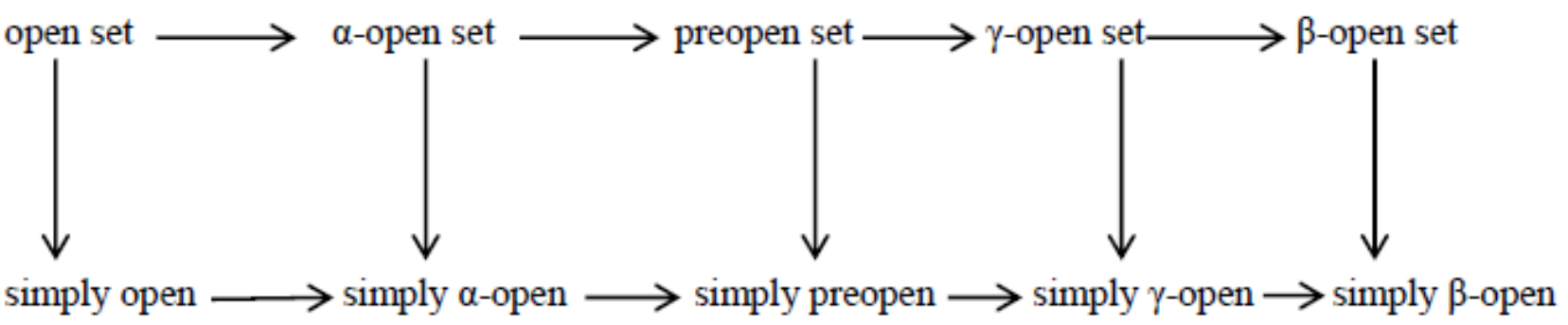

However, the converses are not true in general as shown by the following examples:

Example 4.1 We will consider example 3.4 from [10]. Let $X=\{a, b, c\}, \tau=\{\varnothing,\{a\},\{b, c\}, X\}$ and $\sigma=\{\varnothing,\{a\}, X\}$. Let $f:(X, \tau) \rightarrow(Y, \sigma)$ be the identity function. Clearly, $f$ is simply-continuous but not strongly simply-continuous. Set $V=\{a, b\}$. Note that $V$ is semi-open in $\sigma$ but $V$ is not simply-open in $\tau$.

Example 4.2 Let $X=\{a, b, c\}, \tau=\{\varnothing,\{a\},\{b\},\{a, b\}, X\}$ and $f:(X, \tau) \rightarrow(X, \tau)$ be a function defined as follows: $f(a)=f(b)=a$ and $f(c)=c$. As pointed out in [25], $f$ is not pre $s g$-continuous, thus $f$ is not irresolute. But it is easily checked that $f$ is strongly simply-continuous. 


\section{Proposition 4.3}

1. If $(X, \tau)$ is locally indiscrete, then a function $f:(X, \tau) \rightarrow(Y, \sigma)$ is irresolute if and only if $f$ is simply-continuous.

2. If $(Y, \sigma)$ is globally disconnected, then a function $f:(X, \tau) \rightarrow(Y, \sigma)$ is strongly simply-continuous if and only if $f$ is simply-continuous.

3. If $(X, \tau)$ is strongly irresolvable or, in particular a semi-door space, then every function $f:(X, \tau) \rightarrow(Y, \sigma)$ is strongly simply-continuous.

Example 4.3 Let $X=\{a, b, c, d\}, \tau=\{\varnothing,\{d\},\{a, d\}, X\}$ and $\sigma=\{\varnothing,\{a, d\},\{b, c\}, X\}$. Let $f:(X, \tau) \rightarrow(X, \sigma)$ defined by: $f(a)=a, f(b)=d, f(c)=b, f(d)=c$. Clearly $f$ is simply continuous but not semi-continuous.

From the above proposition, we have the following decomposition of irresoluteness.

Theorem 4.1 For a function $f:(X, \tau) \rightarrow(Y, \sigma)$, the following conditions are equivalent:

1. $f$ is irresolute,

2. $f$ is strongly simply-continuous and pre $s g$-continuous.

Lemma 4.1 For a topological space $(X, \tau)$, we have: a function $f:(X, \tau) \rightarrow(Y, \sigma)$ is $\alpha$-continuous if and only if it is both precontinuous and $D(\alpha, p)$-continuous.

Definition 4.2 A function $f:(X, \tau) \rightarrow(Y, \sigma)$ is called $\alpha$-continuous [22](resp. precontinuous [21]), if $f^{-1}(V)$ is $\alpha$-set (resp. preopen) for each $V \in \sigma$.

Theorem 4.2 For a function $f:(X, \tau) \rightarrow(Y, \sigma)$, the following conditions are equivalent:

1. $f$ is $\alpha$-continuous,

2. $f$ is simply-continuous and precontinuous.

Proof. Evidently, by Lemma 3.1, it is sufficient to prove that every simply-open set belongs to $D(\alpha, p)$. At first we shall show that $B \in D(\alpha, p)$ if and only if $X \backslash B \in D(\alpha, p):$ If $B \in D(\alpha, p)$, then $B \cap \operatorname{Int}(C l(B))=B \cap \operatorname{Int}(C l(\operatorname{Int}(B))) \quad$. Thus we obtain $C l(\operatorname{Int}(B))=C l(\operatorname{Int}(C l(\operatorname{Int}(B))))=C l(C l(B) \cap \operatorname{Int}(C l(\operatorname{Int}(B))))=C l(B \cap \operatorname{Int}(C l(\operatorname{Int}(B))))=C l(B \cap \operatorname{Int}(C l(B)))$ ; consequently $\operatorname{Int}(C l(B))=\operatorname{Int}(C l(\operatorname{Int}(C l(B))))=\operatorname{Int}(C l(\operatorname{Int}(B)))$. Now let us observe that $\operatorname{Int}(C l(\operatorname{Int}(B)))=X \backslash C l(\operatorname{Int}(C l(X \backslash B))) \quad$ and $\quad \operatorname{Int}(C l(B))=X \backslash C l(\operatorname{Int}(X \backslash B)) \quad$. This $\quad$ implies $C l(\operatorname{Int}(C l(X \backslash B)))=C l(\operatorname{Int}(X \backslash B)) \quad$ and consequently $\operatorname{Int}(C l(X \backslash B))=\operatorname{Int}(C l(\operatorname{Int}(C l(X \backslash B))))=\operatorname{Int}(C l(\operatorname{Int}(X \backslash B)))$ So $(X \backslash B) \cap \operatorname{Int}(C l(X \backslash B))=(X \backslash B) \cap \operatorname{Int}(C l(\operatorname{Int}(X \backslash B)))$, which means $X \backslash B \in D(\alpha, p)$.

Secondly, we observe that every open set belongs to $D(\alpha, p)$ and every nowhere dense set belongs to $D(\alpha, p)$. Therefore, by the above fact, every closed set belongs to $D(\alpha, p)$ and every set of the form $X \backslash N$, where $N$ is nowhere dense, also belongs to $D(\alpha, p)$. Then every simply-open set $U \cup N$ is of the form $X \backslash(X \backslash G) \cap(X \backslash N)$, where $(X \backslash G) \cap(X \backslash N)$ belongs to $D(\alpha, p)$ by Lemma 3.1, thus the set $G \cup N$ belongs to $D(\alpha, p)$.

Definition 4.3 A function $f:(X, \tau) \rightarrow(Y, \sigma)$ is called quasi continuous at a point $x \in X$ (see [20]) if fr each neighborhood $U$ of $x$ an each neighborhood open set $G \subseteq U$ such that $f(G) \subseteq V$.

Remark 4.1 It is easy to see that every quasi continuous function is simply continuous. 
Definition 4.4 A function $f:(X, \tau) \rightarrow(Y, \sigma)$ is called almost quasi continuous at a point $x \in X$ (see [5]) if for each neighborhood $V$ of $f(x)$ and each neighborhood $U$ of $x$, the set $f^{-1}(V) \cap U$ is nowhere dense.

Theorem 4.3 A function $f:(X, \tau) \rightarrow(Y, \sigma)$ is quasi continuous iff it is almost quasi continuous and simply continuous.

Proof. Follows directly according to Lemma 7 and Theorem 4 of [5].

Theorem 4.4 Let $f:(X, \tau) \rightarrow(Y, \sigma), g:(Y, \sigma) \rightarrow(Z, \theta)$ be two functions and $g \circ f:(X, \tau) \rightarrow(Z, \theta)$ be the composition of $f$ and $g$. Then the following properties hold: $1 . g \circ f$ is continuous if $f$ is dual simply-continuous and $g$ is simply-continuous,

2. $g \circ f$ is dual simply-continuous if $f$ is continuous and $g$ is dual simply-continuous,

3. $g \circ f$ is strongly semi-continuous if $f$ is dual simply-continuous and $g$ is strongly simply-continuous,

4. $g \circ f$ is strongly semi-continuous if $f$ is dual simply-continuous and $g$ is irresolute,

5. $g \circ f$ is simply-continuous if $f$ is simply-continuous and $g$ is continuous,

6. $g \circ f$ is strongly semi-continuous if $f$ is strongly simply-continuous and $g$ is irresolute,

7. $g \circ f$ is simply-continuous if $f$ is strongly simply-continuous and $g$ is semi-continuous.

\section{New types of simply open sets}

Definition 5.1 A subset $B$ of a topological space $(X, \tau)$ is called simply $\alpha$-open (resp. simply preopen, simply $\gamma$ -open, simply $\beta$-open) if $B=G \cup N$, where $G$ is an $\alpha$-open (resp. preopen, $\gamma$-open, $\beta$-open) set and $N$ is nowhere dense in $(X, \tau)$.

Remark 5.1 From the above definition and Definition 2.1, we have the following implications:

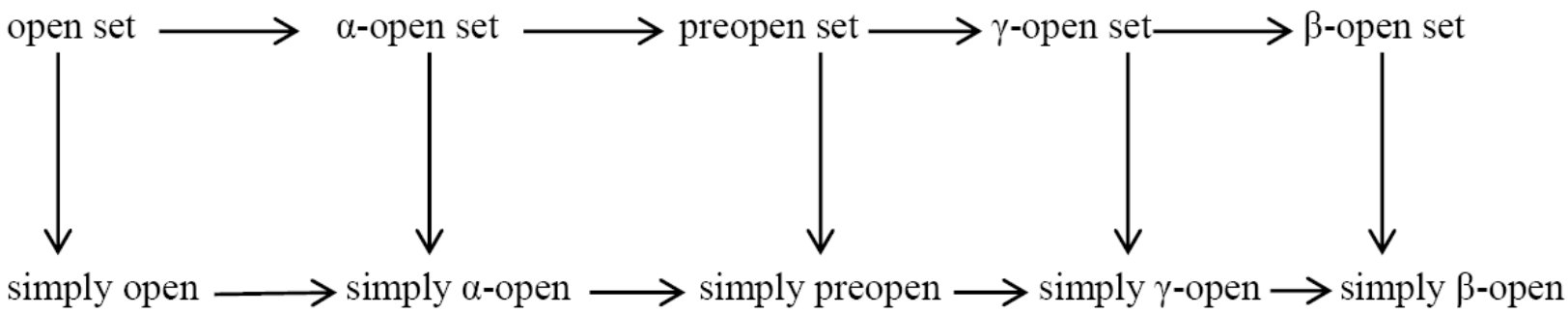

\section{Figure 2:}

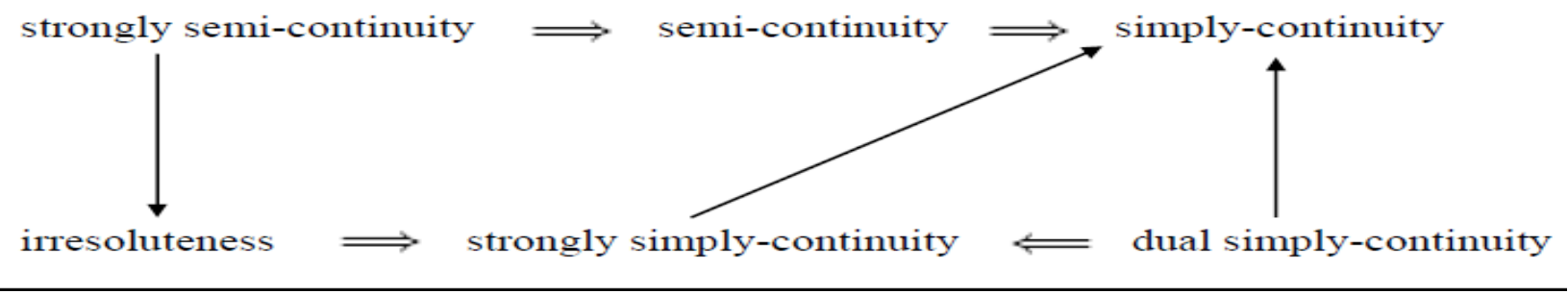

In the remark above, the relationships can not be reversible as the following examples show.

Example 5.1 Let $X=\{a, b, c, d, e\}$ with a topology $\tau$.

(a) If $\tau=\{\varnothing,\{d\},\{a, b\},\{a, b, d\},\{c, d, e\}, X\}$, then

1. $\{c\}$ is simply open but not open,

2. $\{e\}$ is simply $\alpha$-open but not $\alpha$-open, 
3. $\{a, c\}$ is simply preopen but not preopen,

4. $\{a\}$ is simply preopen but not simply $\alpha$-open.

\section{References}

[1] M.E. Abd El-Monsef, R.A. Mahmoud and A.A. Nasef, Strongly semi-continuous functions, Arab Jour. of Phys. and Math. (Iraq), V.(11)(1990), 15-22.

[2] D. Andrijevi c', Some properties of the topology of $\alpha$-sets, Mat. Vesnik 36(1984), 1-10.

[3] P. Bhattacharya and B.K. Lahirir, Semi -generalized closed sets in topology, Indian J.

Math., 29(1987), no. 3, 375-382.

[4] N. Biswas, On some mappings in topological spaces, Bull.Cal. Math. Soc.61(1969),127-135.

[5] J. Borsik and J. Dobous, On decompositions of quasicontinuity, Real Analysis Exchange, Vo. 16 (1990-1991), 292-305.

[6] N. Bourbaki, General Topology, Part 1, Addison Wesly, Rending, Mass., 1966.

[7] M. Caldes and J. Dontchev, G. $\wedge_{s}-$ sets and G. $\vee_{s}-$ sets, Mem.Fac. Sci.Kochi. Univ. (Math.) 21(2000), 21-30.

[8] C. Chattopadhyay and U.K. Roy, $\delta$-sets, irresolvable space, Math., Slovaca, 42(1992), no.3, 371-378.

[9] S.G. Crossley and S.K. Hildebrand, semi-topological properties, Fund. Math, 74(1972), 233-254.

[10] J. Dontchev, The characterization of some peculiar topological spaces via A - and B -sets, Acta Math. Hunger., 67(3)(1995), 67-71.

[11] J. Dontchev and M. Ganster, A decomposition of irresoluteness, Acta Math. Hungarica 77(1-2)(1997), 41-46.

[12] A.G. El'kin, Decomposition of spaces, Soviet Math. Dokl., 10(1969), 521-525.

[13] J. Ewert, On quasi-continuous and cliquish maps with values in uniform spaces, Bull. Acad. Polon. Sci., 32(1984), 81-88.

[14] J. Foran and P. Liebnitz, A characterization of almost resolvable spaces, Rend. Circ. Mat. Palermo, Serie II, Tomo XL (1991), 136-141.

[15] M. Ganster, I.L. Reilly and M.K. Vamanamurthy, Remarks on locally closed sets, Math. Pannonica, 3(2)(1992), 107-113.

[16] D.S Jankoivi c' , On locally irreducible spcaes, Ann. Soc. Sci. Bruxelles Ser. I, 97(1983), no.2, 59-72.

[17] K. Kuratowski, Topology, Vol. I, Academic press, New york, 1966.

[18] N. Levine, semi-open set and semi -continuty in topological spaces, Amr. Math. Monthly, 70(1963), 36-41.

[19] S.N. Maheshwari and R. Prasad, On $R_{0}$ - spaces, Portugal Math., 34(1975), 213-217.

[20] S. Marcus, Suvless functions quasicontinouns au sens de S. Kempists, Collage. Math. 8(1961), 47-53.

[21] A.S. Mashhour, M.E. Abd El-Monsef and S.N. El-Deeb, On precontinuous and weak precontinuous mappings, Proc. Math. Phys. Soc. Egypt, 53(1982), 47-53.

[22] A.S. Mashhour, I.A. Hasanein and S.N. El-Deeb, $\alpha$-continous and $\alpha$-open mappings, Acta Math. Hungar., 47(1983), 213-218.

[23] A. Neubrunnová, On transfinite sequences of certain types of functions, Acta Fac.Rer. Natur. Univ. Com. Math. 30(1975), 121-126.

[24] O. Njåstad, On some classes of nearly open sets, Pacific J. Math., 15(1965), 961-970.

[25] T. Noiri, Semi-normal spaces and some functions, Acta Math. Hungar,65(3)(1994),305-311.

[26] M.H. Stone, Applications of the Theory of Boolean Rings to General Topology, TAMS,41(1937),375-381.

[27] A.H. Stone, Absolutely FG-spaces, Proc. Amer. Math. Soc., 80(1980), 515-520.

[28] P. Sundaram and K. Balachandran, Semi -generalized locally closed sets in topological spaces, preprint.

[29] J.P. Thomas, Maximal connected topologies, J. Austral Math. Soc. Ser.A,8(1968), 700-705.

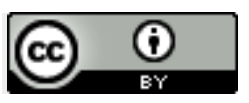

This work is licensed under a Creative Commons Attribution 4.0 International License. 